SLAC-PUB-12044 Revised

November 21, 2006

\title{
Spin Rotation and Energy Compression in the ILC Linac-to-Ring Positron Beamline*
}

\author{
Yuri K. Batygin \\ SLAC, Stanford University, Stanford, CA 94309
}

\begin{abstract}
The Linac-to Ring (LTR) beamline for positron beam injection into the damping ring at an energy of $5 \mathrm{GeV}$ is considered. The beamline performs spin rotation and energy compression of the positron beam extracted from the booster linac. The lattice includes four 90-deg phaseadvance FODO cells with bending magnets placed between them, a solenoid and RF structure. Basic parameters and optimal configuration details are discussed. An analytical treatment of the beamline is performed.
\end{abstract}

To be published in Nuclear Instruments and Methods in Physics Research A

*Work supported by Department of Energy contract DE-AC02-76SF00515 


\section{Introduction}

Operation of the future International Linear Collider (ILC) with polarized electron and positron beams will significantly improve the precise measurements in new physics. Polarized positrons will be generated by the method proposed by V.E.Balakin and A.A.Michailichenko in 1979 [1]. In this scheme, a high-energy electron beam passes through a helical undulator and produces circularly polarized photons, which then impinge on a target and produce longitudinally polarized positrons. Positrons are generated and captured by the collection system including an Adiabatic Matching Device, pre-accelerator, long beam transport line and $5 \mathrm{GeV}$ booster linac. A positron Linac-to-Ring beamline is a part of a Linear Collider for injection of a 5 $\mathrm{GeV}$ positron beam extracted from positron booster linac, into the positron damping ring. The lattice performs both rotation of the polarization vector from longitudinal to vertical position, and beam energy compression to match the beam with longitudinal acceptance of the damping ring. The acceptance of the positron damping ring is restricted by a full energy spread of $\Delta \gamma / \gamma=1 \%$, bunch length of $4.5 \mathrm{~cm}$, and normalized beam emittance of $\varepsilon_{x}+\varepsilon_{y} \leq 0.09 \pi \mathrm{m} \operatorname{rad}$ [2].

\section{Fundamental Parameters}

In the proposed ILC injector, positrons are longitudinally polarized. As the damping rings require a vertically polarized beam, the polarization vector has to be changed from longitudinal to vertical. Rotation of the polarization vector is performed in two stages [3]: (i) rotation from longitudinal to the radial position in the median plane of bending magnets and (ii) a rotation from radial to the vertical position in a solenoid. The spin precession angle, $\varphi$, in a bending arc is given by:

$$
\varphi=G \gamma \theta
$$

where $\theta$ is the bending arc angle, $G=0.001159652$ is the anomalous magnetic moment of positron, and $\gamma$ is the particle energy. The spin precession angle should be set as $\varphi=90^{\circ}+\mathrm{n} 180^{\circ}$, where $\mathrm{n}$ is the integer, to ensure further spin rotation from horizontal to vertical position. Therefore, for a $5 \mathrm{GeV}$ beam the arc bending angle is [4]:

$$
\theta=\frac{\varphi}{G \gamma}=7.93^{\circ}+n 15.87^{\circ}
$$

The required solenoid strength for rotation of the polarization vector through an angle $\varphi=90^{\circ}$ is defined as [4]:

$$
\int B_{z} d z=\frac{p \varphi}{e(1+G)}=26.18 \text { Tesla } m
$$

Apart from spin rotation, bending magnets are also used for bunch rotation in longitudinal phase space for beam matching with acceptance of the damping ring [3]. A sequence of bending magnets generate bunch decompression due to a non-zero value of $R_{56}$, the transfer matrix element responsible for coupling of longitudinal particle motion with energy spread within the beam: 


$$
l_{b}=l_{b, o}+R_{56} \frac{\Delta \gamma}{\gamma}
$$

where $l_{b, o}$ is the bunch length in front of the structure, and $l_{b}$ is the bunch length at the exit of the arc. After lengthening of the bunch, an RF voltage is applied to rotate beam in longitudinal phase space to match the beam with longitudinal acceptance of the positron damping ring. The energy change within the bunch is given by $d \gamma / d z=e \operatorname{Esin} \varphi_{R F} /\left(m c^{2}\right)$, where $E$ is the amplitude of RF field and $\varphi_{R F}$ is the RF phase of the particle. Adjusting the RF field to zero in the center of the bunch, the required value of the RF voltage to transform the beam is:

$$
E L_{R F}=\frac{m c^{2}}{e} \frac{\Delta \gamma}{2 \sin \left(\pi \frac{l_{b}}{\lambda}\right)} .
$$

Eqs. (2.1) - (2.5) define basic beamline parameters to perform both spin rotation and bunch matching with the positron damping ring.

\section{Beamline Analysis}

Consider a FODO quadrupole structure with bending magnets between quadrupoles, and phase advance $\mu$ per structure period. The structure must be dispersion-free to avoid any emittance growth associated with energy spread within the bunch. It is well known, that the dispersion is suppressed by $m=\pi / \mu$ FODO cells with bending magnets placed between quadrupoles [5, p.61]. To make a structure shorter, it is reasonable to select a small value of $m$, which corresponds to large values of $\mu$. Choosing $m=2$ requires a phase advance of $\mu=90^{\circ}$. Since the structure starts with a zero dispersion region and ends with a zero dispersion region, the arc should contain 4 FODO cells, or 8 quadrupoles and 8 bending magnets. In this case, the bending angle of each magnet, $\alpha$, is equal to one-eight of the total arc angle, $\theta$, Eq. (2.2):

$$
\alpha=\frac{\theta}{8}
$$

Let us analyze the properties of the structure in using the thin-lens approximation, assuming the length of each quadrupole is negligible with respect to the length of bending magnets (see Appendix). Thin quadrupole lens with gradient $G$ and length $d$ is characterized by the focal length $F$ given by:

$$
F=\frac{p}{e G d}
$$

where $p=m c \beta \gamma$ is the momentum of the particles. The focal length, $F$, is related to the FODO period, $L_{p}$, by the equation $[5, \mathrm{p} .60]$ :

$$
F=\frac{L_{p}}{4 \sin \frac{\mu}{2}} .
$$


Since one period of the FODO structure contains 2 quadrupoles and 2 bending magnets, and the bending length is equal to half of the FODO period, $\rho \alpha=L_{p} / 2$, (where $\rho$ is the bending radius) then Eq. (3.3) can be written as:

$$
F=\frac{\rho \alpha}{2 \sin \frac{\mu}{2}}=\frac{\rho \alpha}{\sqrt{2}}
$$

Combining Eqs. (3.2), (3.4) one obtains the following expression connecting quadrupole and bending magnet parameters:

$$
\rho \alpha=2 \sin \frac{\mu}{2} \frac{p}{e G d}
$$

The value of the $R_{56}$ element of the transfer matrix, responsible for bunch decompression, is given by (see Appendix, Eq. (A.11))

$$
R_{56}=-\frac{46}{3} \rho \alpha^{3}
$$

Furthermore, the value of $R_{56}$ is determined by the required specifications of the structure. From Eq. (3.6), the bend length is $\rho \alpha=(3 / 46)\left|R_{56}\right| / \alpha^{2}$, and thus the larger $\alpha$, the shorter system. The maximum beam size, $r_{\text {max }}$, is determined by the maximum value of beta-function of the structure, $\beta_{\max }=L_{p}(1+\sin (\mu / 2)) / \sin \mu$, normalized beam emittance, $\varepsilon$, momentum dispersion, $R_{16}$, and beam energy spread:

$$
r_{\max }=\sqrt{\beta_{\max } \frac{\varepsilon}{\gamma}+\left(\frac{1}{2} \frac{\Delta \gamma}{\gamma} R_{16 \max }\right)^{2}}
$$

Maximum dispersion of the structure is achieved in the middle of the arc and is given by (see Appendix, Eq. (A.10)):

$$
R_{16, \max }=\rho \alpha^{2}(4+\sqrt{2}) \text {. }
$$

Substitution of Eqs. (3.8), (3.6) into Eq. (3.7) gives for the maximum beam size:

$$
r_{\text {max }}=\frac{1}{\alpha} \sqrt{\frac{3}{23} R_{56} \frac{\left(1+\sin \frac{\mu}{2}\right)}{\sin \mu} \frac{\varepsilon}{\gamma}+\left[\frac{3}{92}(4+\sqrt{2}) R_{56} \frac{\Delta \gamma}{\gamma}\right]^{2}}
$$

From Eq. (3.9), the maximum beam radius is smaller for larger values of $\alpha$ as it is inversely proportional to $\alpha$. Therefore, larger values of $\alpha$ are preferable to keep the system compact as possible. However, $\alpha$ is limited by beam depolarization in the arc. In presence of energy spread in the beam, the polarization loss is [6]:

$$
\delta\left(\frac{\Delta P}{P}\right)=1-\cos (\delta \varphi),
$$


where $\delta \varphi=\varphi(\delta \gamma / \gamma)$ is the deviation in spin rotation angle from the nominal value $\varphi$ because of energy deviation, $\delta \gamma$. Assuming the energy distribution within the bunch is Gaussian with rms value of $\sigma_{E}$ :

$$
\frac{d N}{d(\delta \gamma)}=\frac{1}{\sqrt{2 \pi} \sigma_{E}} \exp \left[-\frac{(\delta \gamma)^{2}}{2 \sigma_{E}^{2}}\right]
$$

the depolarization within the bunch is given by integration of the product of expressions given in Eqs. (3.10), (3.11):

$$
\frac{\Delta P}{P}=\frac{1}{\sqrt{2 \pi} \sigma_{E}} \int_{-\infty}^{\infty}\left[1-\cos \left(\varphi \frac{\delta \gamma}{\gamma}\right)\right] \exp \left[-\frac{(\delta \gamma)^{2}}{2 \sigma_{E}^{2}}\right] d(\delta \gamma) .
$$

Integration of Eq. (3.12) enables the beam depolarization to be obtained:

$$
\frac{\Delta P}{P}=1-\exp \left(-\varphi^{2} \frac{\sigma_{E}^{2}}{2 \gamma^{2}}\right) \approx \frac{1}{2}\left(\varphi \frac{\sigma_{E}}{\gamma}\right)^{2}
$$

The above Eq. (3.13) shows that an increase of arc angle $\theta=\varphi /(G \gamma)$ results in larger beam depolarization for a fixed value of beam energy spread.

\section{Beamline Parameters}

Table 1 and Fig. 1 contain results of design of $5 \mathrm{GeV}$ positron LTR beamline. The transfer matrix $R_{56}=1 \mathrm{~m}$ and arc angle $\theta=55^{\circ}$ were selected to provide sufficient compression of the bunch with energy spread of $\Delta \gamma / \gamma=3.5 \%$ into $\Delta \gamma / \gamma=1 \%$. The half-period of the structure, from Eq. (3.6) is evaluated as:

$$
\rho \alpha=\frac{3}{46} \frac{R_{56}}{\alpha^{2}}=4.44 m
$$

Also, the strength of quadrupoles are given by Eq. (3.5)

$$
G d=2 \sin \frac{\mu}{2} \frac{p}{e \rho \alpha}=5.39 \mathrm{Tm} .
$$

Taking the length of quadrupoles as $d=0.3 \mathrm{~m}$, the gradients are $G=17.77 \mathrm{~T} / \mathrm{m}$. In practice, the optimized quadrupole gradients are slightly different from this value because of the presence of dipole magnets which provide additional radial focusing (see Table 1).

Bending magnet lengths are selected to be shorter than that given by Eq. (4.1) to provide some space between bending magnets and quadrupoles. The distance between centers of quadrupoles are defined by Eq. (4.1). Taking the value of bending field as $B=1.18 \mathrm{~T}$, the length of bending magnets is:

$$
L_{b}=\frac{p \alpha}{e B}=1.7 \mathrm{~m}
$$


From Eq. (4.3), the drift space between bending magnets and quadrupoles is

$$
L=\frac{1}{2}\left(\rho \alpha-L_{b}-d\right)=1.21 \mathrm{~m}
$$

A bending arc is followed by an RF structure with gradient of $30 \mathrm{MeV} / \mathrm{m}$ and length of $6 \mathrm{~m}$ providing the required RF voltage of $E L_{R F}=180 \mathrm{MeV}$. After an RF structure, a solenoid with the field of $3.14 \mathrm{~T}$ and length of $8.32 \mathrm{~m}$ is used to provide $90^{\circ}$ spin rotation from transverse to vertical position according to Eq. (2.3).

\section{Positron Beam Capture and Polarization}

An estimation of polarized positron beam capture and expected polarization in the ILC injector prior to the LTR beamline was performed in Ref. [7] using the code BEAMPATH [8]. Based on these results Fig. 2 illustrates positron beam distribution after on-crest acceleration of positrons up to the energy of $5 \mathrm{GeV}$. After the particles impinge on the target they are meant to pass through an Adiabatic Matching Device, and then accelerated up to an energy of $5 \mathrm{GeV}$. The particles captured in the head of the bunch are more polarized than those in the tail of the bunch because of the initial correlation between polarization and energy of particles generated at the target. Particle tracking was accompanied with an integration of the Thomas-BMT equation, describing the precession of the spin vector $\vec{S}$ :

$$
\frac{d \vec{S}}{d t}=\frac{e \vec{S}}{m \gamma} x\left[(1+G \gamma) \vec{B}_{\perp}+(1+G) \vec{B}_{I I}+\left(G \gamma+\frac{\gamma}{1+\gamma}\right) \frac{\overrightarrow{E x} \vec{\beta}}{c}\right]
$$

where $\vec{E}$ is the electrical field, and $\vec{B}_{\perp}$ and $\vec{B}_{I I}$ are components of the magnetic field perpendicular and parallel to particle velocity. The beam polarization is defined as an average of the product of the spin components $S_{x}, S_{y}, S_{z}$ and the value of polarization, $P$, summed over all positrons:

$$
\left\langle P_{x}\right\rangle=\frac{1}{\mathrm{~N}} \sum_{i=1}^{\mathrm{N}} S_{x}^{(i)} P^{(i)}, \quad\left\langle P_{y}\right\rangle=\frac{1}{\mathrm{~N}} \sum_{i=1}^{\mathrm{N}} S_{y}^{(i)} P^{(i)}, \quad\left\langle P_{z}\right\rangle=\frac{1}{\mathrm{~N}} \sum_{i=1}^{\mathrm{N}} S_{z}^{(i)} P^{(i)}
$$

The value of positron capture is defined as a fraction of captured positrons within $6 \mathrm{D}$ acceptance of the damping ring with respect to number of positrons produced at the target. The positron capture of the beam illustrated in Fig. 2 can reach $32 \%$ for $\varepsilon_{x}+\varepsilon_{y}=0.09 \pi \mathrm{m}$ rad with longitudinal polarization of $\left\langle P_{z}\right\rangle=0.57$.

The effect of LTR is illustrated in Figs. 3-6. The particle distribution before LTR (see Fig. 3 ) is selected to be off-crest with energy spread of $\Delta E / E \leq 3.5 \%$ to insure a sufficient increase in the number of captured positrons. Fig. 4 illustrates distribution of positrons after the $55^{\circ}$ arc. The positron distribution is tilted in longitudinal phase space because of bunch decompression in the arc whilst the energy spread is left unchanged.

Applying an RF voltage results in a flattening of the beam distribution in longitudinal phase space (see Fig. 5). After the LTR, the beam is matched to the acceptance of the damping ring. Positrons captured with an energy resolution of $\Delta E / E=1 \%$ can reach $50 \%$ with an average beam polarization of $\left\langle P_{y}\right\rangle=0.53$. The acceptance of more particles is accompanied by 
decreasing beam polarization because the additional accepted positrons from the tail of the bunch are less polarized than that in the head of the bunch. Fig. 6 illustrates variation of components of the average beam polarization, Eq. (5.2) along the structure. The beam is longitudinally polarized at the entrance of the structure and this is transformed into a beam with vertical polarization.

The effect of beam depolarization in the arc may be estimated from Eq. (3.13). The full width energy spread of the transformed bunch is $\Delta \gamma / \gamma=3.5 \%$ and the corresponding rms energy spread is approximately $\sigma_{E} / \gamma \approx(1 / 4) \Delta \gamma / \gamma=0.875 \%$. For a spin rotation angle of $\varphi=7 / 2 \pi$, the beam depolarization is $\triangle P / P=4.610^{-3}$.

\section{Conclusion}

The ILC beamline for positron beam injection into the damping ring has been considered. The beamline contains four $90^{\circ}$ FODO cells accompanied by bending magnets in between the quadrupoles. The arc magnets provide spin rotation in the median plane and bunch decompression. The solenoid performs spin rotation in vertical direction and the RF station matches the longitudinal phase space of the beam to acceptance of the damping ring. Tracking analysis indicates that utilization of LTR increases the positron capture from $32 \%$ to $50 \%$ whilst the beam polarization drops from 0.57 to 0.53 as a consequence of accepting additional positrons with a low polarization.

\section{Acknowledgements}

The author is indebted to Dr R.M. Jones for carefully reading the manuscript and for providing critical comments. 


\section{References}

1. V.E.Balakin, A.A.Michailichenko, “The Conversion System for Obtaining High Polarized Electrons and Positrons", INP 79-85, Novosibirsk, 1979.

2. ILC Baseline Configuration Document, http://www.linearcollider.org/wiki/doku.php?id=bcd:bcd home

3. Zeroth-Order Design Report for the Next Linear Collider, LBNL-5424, SLAC-474, UCRLID-124161, UC-414, 1996.

4. K. Moffeit, M.Woods, P.Schuler, K.L. Moenig, P.Bambade, "Spin Rotation Schemes at the ILC for Two Interaction Regions and Poistron Polarization with Both Helicities", SLAC-TN05-045, 2005.

5. Handbook of Accelerator Physics and Engineering Edited by A.W.Chao and M.Tigner, World Scientific, 1999.

6. P.Emma, T.Limberg, R.Rossmanith, "Depolarization in the SLC Collider Arcs“, SLAC-PUB6527, 1994.

7. Y.K.Batygin, Positron Collection in Linear Collider, 2005 ALCPG \& ILC Workshop, Snowmass, SLAC-PUB-11590, 2005.

8. Y.K.Batygin, NIM-A 539, p. p. 455 - 489, 2005. 
Table 1. Parametes of Beamline.

Arc Quadrupoles

Number

8

Gradient $\quad 17.8 \mathrm{~T} / \mathrm{m},-18.18 \mathrm{~T} / \mathrm{m}$

Length

$0.3 \mathrm{~m}$

Matching Quadrupoles

Number

Gradient

9

Length

$14 \ldots 33 \mathrm{~T} / \mathrm{m}$

$0.2 \ldots 0.6 \mathrm{~m}$

Bending Magnets

Number

Bending angle

8

Length

$6.94^{\circ}$

Field

$1.7 \mathrm{~m}$

$1.188 \mathrm{~T}$

RF Structure

Gradient

$30 \mathrm{MV} / \mathrm{m}$

Length

$6 \mathrm{~m}$

Solenoid

Field

$3.15 \mathrm{~T}$

Length

$8.32 \mathrm{~m}$ 
Appendix

\section{Transformation Matrix for Beamline Arc}

Consider a sequence of quadrupole lenses with bending magnets between them. The $x$ and $y$ motion is not coupled in linear approximation, and this enables us to use a matrix method for $\mathrm{x}$ $\mathrm{z}$ motion in the form of

$$
\left|\begin{array}{c}
x \\
x^{\prime} \\
l \\
\delta
\end{array}\right|=\left|\begin{array}{llll}
R_{11} & R_{12} & R_{15} & R_{16} \\
R_{21} & R_{22} & R_{25} & R_{26} \\
R_{51} & R_{52} & R_{55} & R_{56} \\
R_{61} & R_{62} & R_{65} & R_{66}
\end{array}\right|\left|\begin{array}{c}
x_{o} \\
x_{o}^{\prime} \\
l_{o} \\
\delta
\end{array}\right|,
$$

We divide each quadrupole lens into two half-lenses (see Fig. 7). The matrices of focusing, $M_{F}$, and defocusing, $M_{D}$, (half thin quadrupole) are given by

$$
M_{F}=\left|\begin{array}{rrrr}
1 & 0 & 0 & 0 \\
-\frac{1}{f} & 1 & 0 & 0 \\
0 & 0 & 1 & 0 \\
0 & 0 & 0 & 1
\end{array}\right|, \quad M_{D}=\left|\begin{array}{llll}
1 & 0 & 0 & 0 \\
\frac{1}{f} & 1 & 0 & 0 \\
0 & 0 & 1 & 0 \\
0 & 0 & 0 & 1
\end{array}\right| .
$$

where $f$ is the focal length of half quadrupole:

$$
f=2 \frac{p}{e G d}
$$

The matrix of a bending magnet:

$$
M_{\text {bend }}=\left|\begin{array}{cccc}
\cos \alpha & \rho \sin \alpha & 0 & \rho(1-\cos \alpha) \\
-\frac{1}{\rho} \sin \alpha & \cos \alpha & 0 & \sin \alpha \\
-\sin \alpha & -\rho(1-\cos \alpha) & 1 & -\rho(\alpha-\sin \alpha) \\
0 & 0 & 0 & 1
\end{array}\right| .
$$

is replaced by the matrix form, where only first non-vanishing terms are retained:

$$
M_{B}=\left|\begin{array}{cccc}
1 & \rho \alpha & 0 & \rho \frac{\alpha^{2}}{2} \\
-\frac{\alpha}{\rho} & 1 & 0 & \alpha \\
-\alpha & -\rho \frac{\alpha^{2}}{2} & 1 & -\rho \frac{\alpha^{3}}{6} \\
0 & 0 & 0 & 1
\end{array}\right| .
$$

The matrix of the complete half-period, including half of focusing quadrupole, bending magnet, and half of defocusing qudrupole, is obtained as: 


$$
M_{D} M_{B} M_{F}=\left|\begin{array}{cccc}
1-\frac{\rho \alpha}{f} & \rho \alpha & 0 & \rho \frac{\alpha^{2}}{2} \\
-\rho \frac{\alpha}{f^{2}} & 1+\frac{\rho \alpha}{f} & 0 & \frac{\rho \alpha^{2}}{2 f}+\alpha \\
\frac{\rho \alpha^{2}}{2 f}-\alpha & -\rho \frac{\alpha^{2}}{2} & 1 & -\rho \frac{\alpha^{3}}{6} \\
0 & 0 & 0 & 1
\end{array}\right| .
$$

In derivations of Eq. (A.6) it was assumed that $1+(f / \rho)^{2} \approx 1$. The matrix of a single period $M_{\text {period }}=M_{F} M_{B} M_{D} M_{D} M_{B} M_{F}$ is obtained by multiplying the two expressions, Eq. (A.6), with the opposite signs of substituted for the terms containing a focal length $f$ :

$$
M_{\text {period }}=\left|\begin{array}{cccc}
1-2\left(\frac{\rho \alpha}{f}\right)^{2} & 2 \rho \alpha\left(1+\frac{\rho \alpha}{f}\right) & 0 & \rho \alpha^{2}\left(2+\frac{\rho \alpha}{f}\right) \\
-\frac{2 \rho \alpha}{f^{2}\left(1-\frac{\rho \alpha}{f}\right)} & 1-2\left(\frac{\rho \alpha}{f}\right)^{2} & 0 & \alpha\left[2-\frac{\rho \alpha}{f}-\left(\frac{\rho \alpha}{f}\right)^{2}\right] \\
-\alpha\left[2-\frac{\rho \alpha}{f}-\left(\frac{\rho \alpha}{f}\right)^{2}\right] & -\rho \alpha^{2}\left(2+\frac{\rho \alpha}{f}\right) & 1 & -\rho \alpha^{3}\left(\frac{4}{3}+\frac{\rho \alpha}{2 f}\right) \\
0 & 0 & 0 & 1
\end{array}\right| .
$$

We now take into account the details of the structure described by Eq. (3.4)

$$
\frac{\rho \alpha}{f}=\sin \frac{\mu}{2}=\frac{1}{\sqrt{2}}
$$

Under this constraint, matrix, Eq. (A.7) is written as:

$$
M_{\text {period }}=\left|\begin{array}{cccc}
0 & \rho \alpha \sqrt{2}(1+\sqrt{2}) & 0 & \rho \alpha^{2}\left(2+\frac{1}{\sqrt{2}}\right) \\
-\frac{1}{f}(\sqrt{2}-1) & 0 & 0 & \alpha\left(\frac{3-\sqrt{2}}{2}\right) \\
-\alpha\left(\frac{3-\sqrt{2}}{2}\right) & -\rho \alpha^{2}\left(2+\frac{1}{\sqrt{2}}\right) & 1 & -\rho \alpha^{3}\left(\frac{4}{3}+\frac{1}{2 \sqrt{2}}\right) \\
0 & 0 & 0 & 1
\end{array}\right| .
$$

Multiplying the two cell matrixes, Eq. (A.9), enables us to obtain a matrix describing half the system, $M_{1 / 2}=M_{\text {period }} M_{\text {period }}$ :

$$
M_{1 / 2}=\left|\begin{array}{cccc}
-1 & 0 & 0 & \rho \alpha^{2}(4+\sqrt{2}) \\
0 & -1 & 0 & 0 \\
0 & -\rho \alpha^{2}(4+\sqrt{2}) & 1 & -\frac{23}{3} \rho \alpha^{3} \\
0 & 0 & 0 & 1
\end{array}\right| .
$$


Finally, taking the product of two half-system matrixes, Eq. (A.10), allows us to evaluate the matrix of the complete structure, $M_{a r c}=M_{1 / 2} M_{1 / 2}$

$$
M_{a r c}=\left|\begin{array}{cccc}
1 & 0 & 0 & 0 \\
0 & 1 & 0 & 0 \\
0 & 0 & 1 & -\frac{46}{3} \rho \alpha^{3} \\
0 & 0 & 0 & 1
\end{array}\right| .
$$


a)

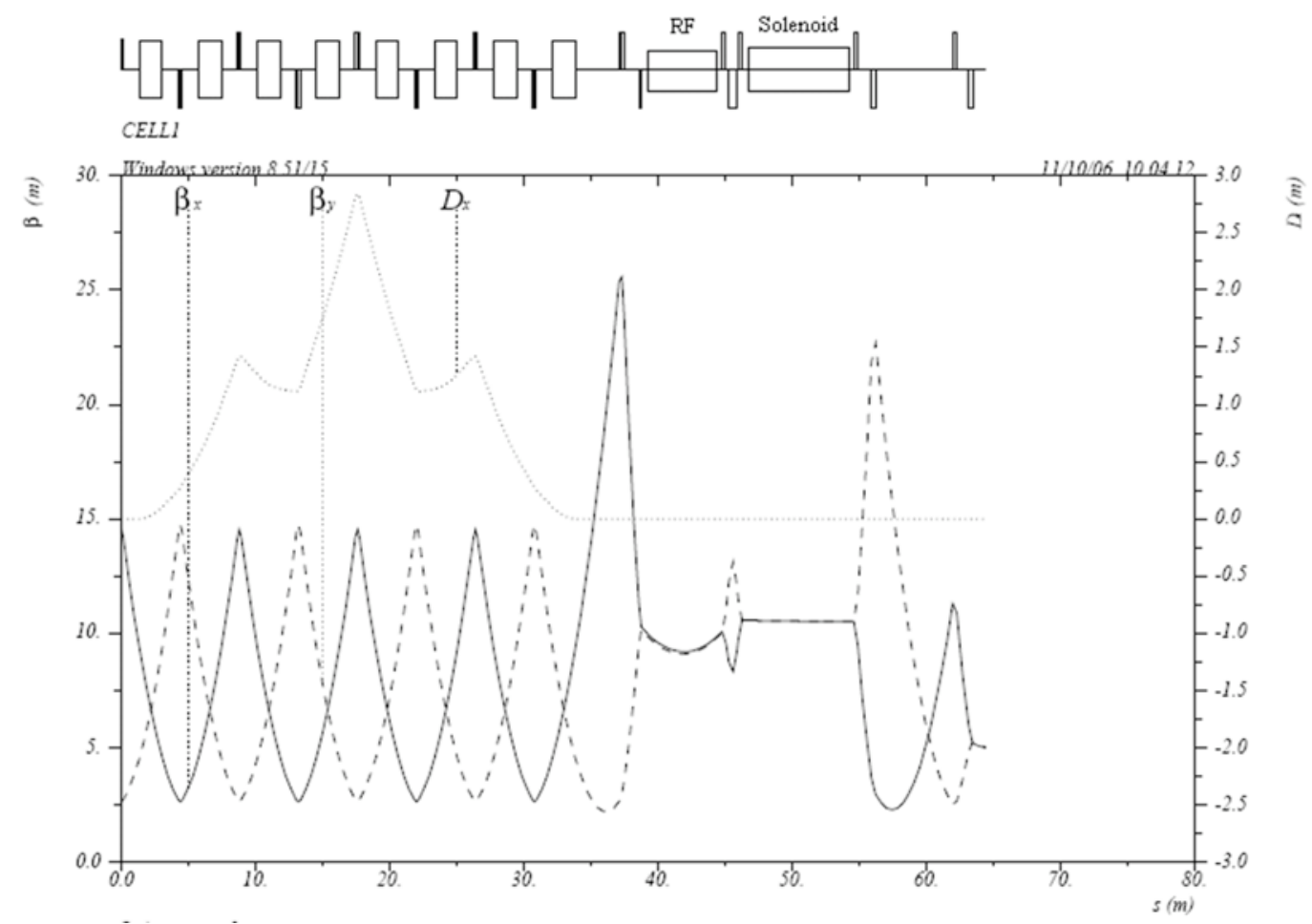

b)

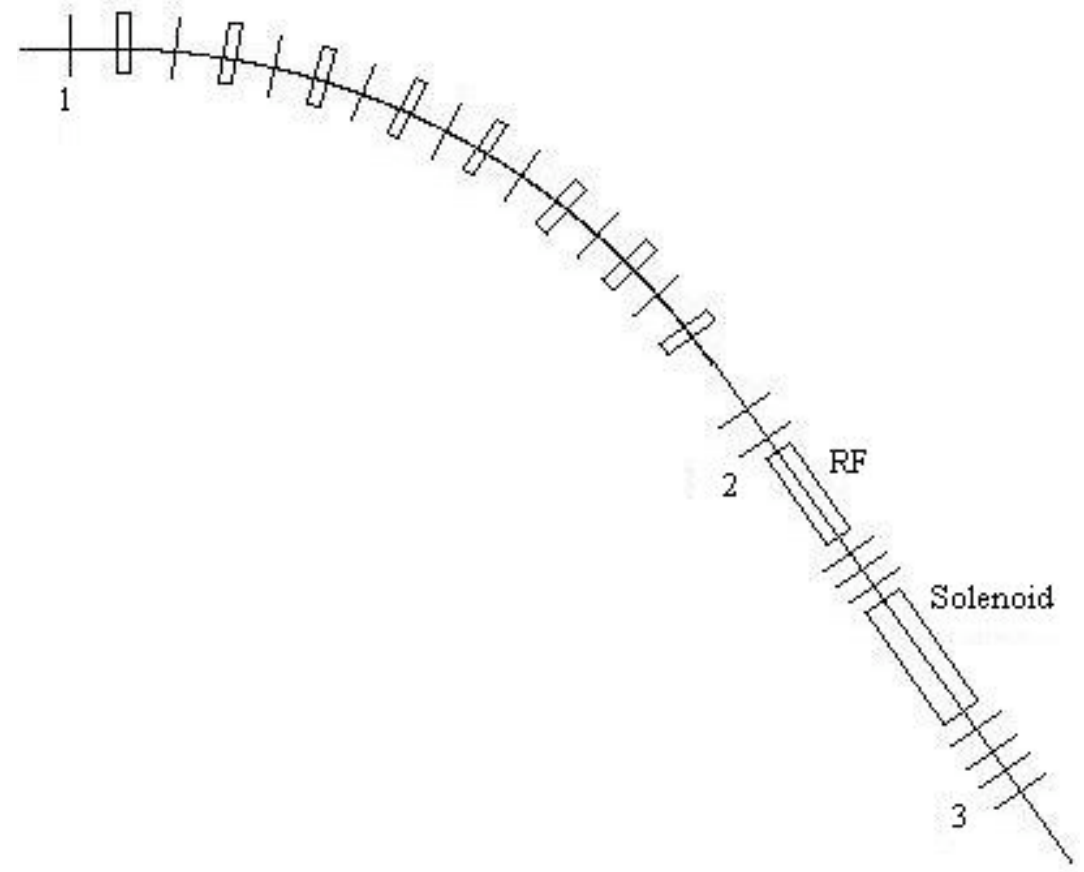

Fig. 1. (a) Beamline lattice, (b) beamline layout. Numbers correspond to 1 - entrance at the beamline, (see Fig. 3), 2 - position after arc (see Fig. 4), and 3 - exit of beamlline (see Fig. 5). 

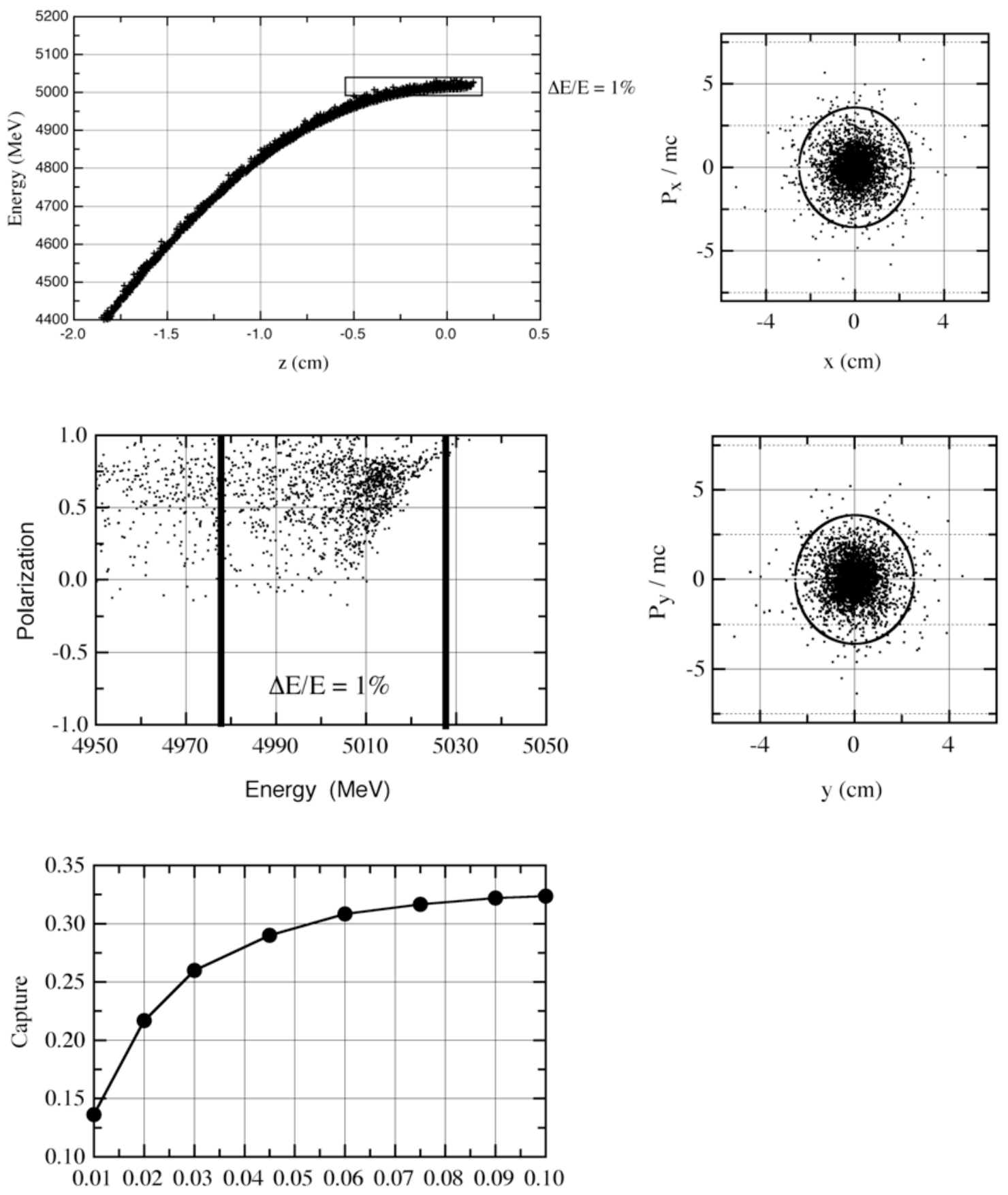

$$
\varepsilon_{\mathrm{X}}+\varepsilon_{\mathrm{y}}(\pi \mathrm{m} \mathrm{rad})
$$

Fig. 2. Positron beam distribution and capture within energy resolution of $\Delta \mathrm{E} / \mathrm{E} \leq 1 \%$ without LTR. Positron beam polarization is 0.57 . Ellipses comprise phase space area of $0.09 \pi \mathrm{m}$ rad. 

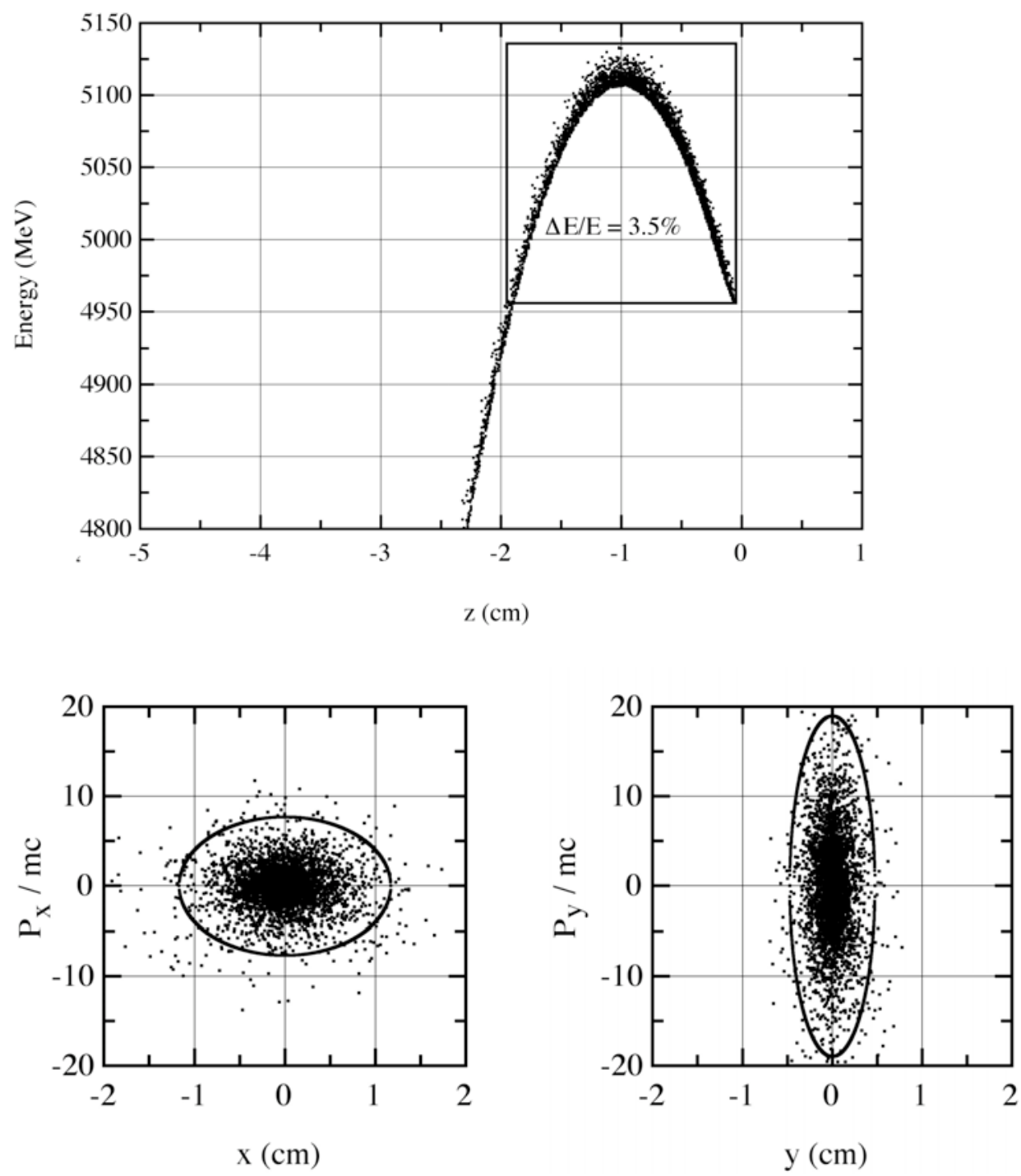

Fig. 3. Distribution of positrons at the entrance of LTR beamline. 

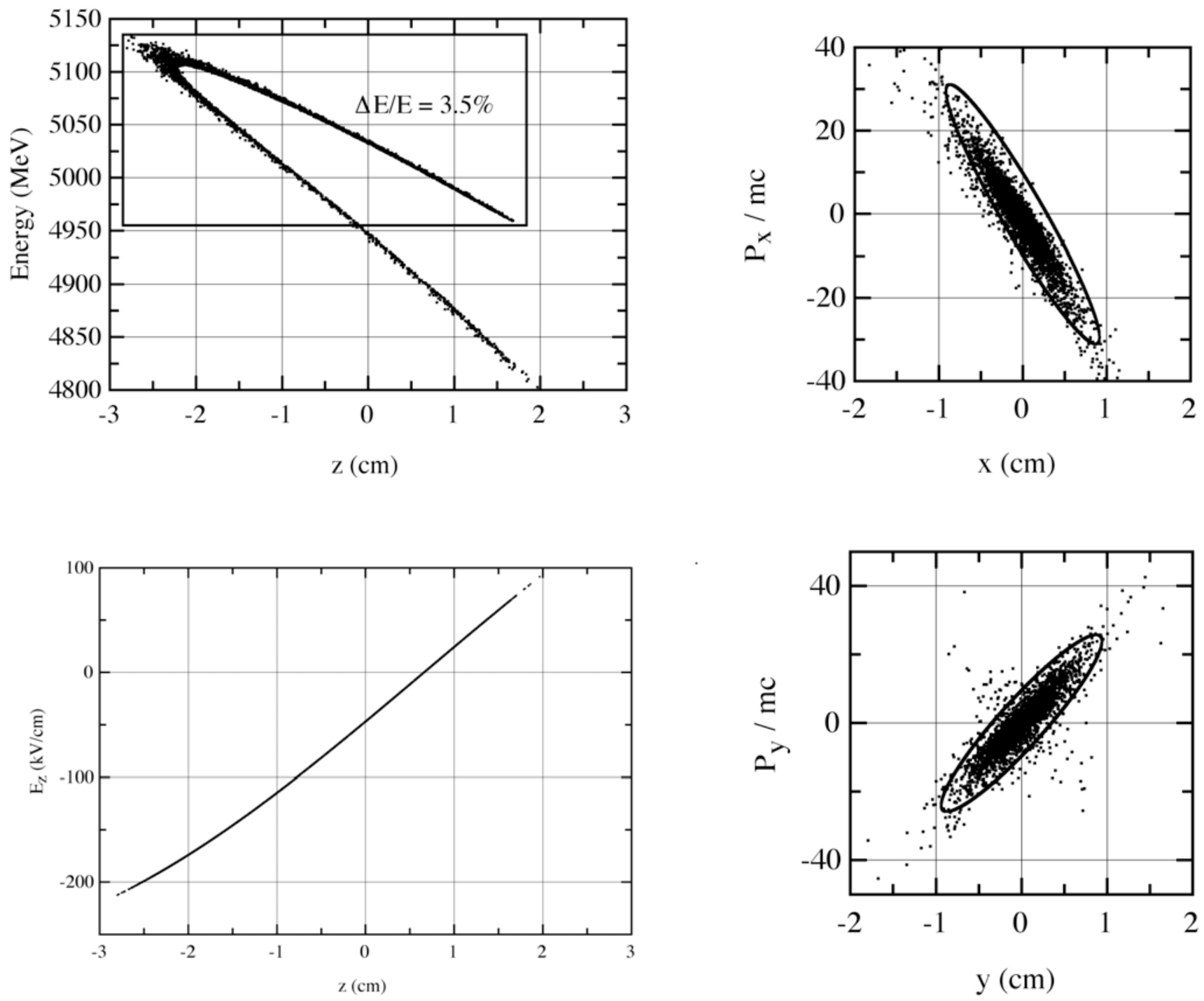

Fig. 4. Distribution of positrons after $55^{\circ}$ bending arc and RF field acting at the particles. 

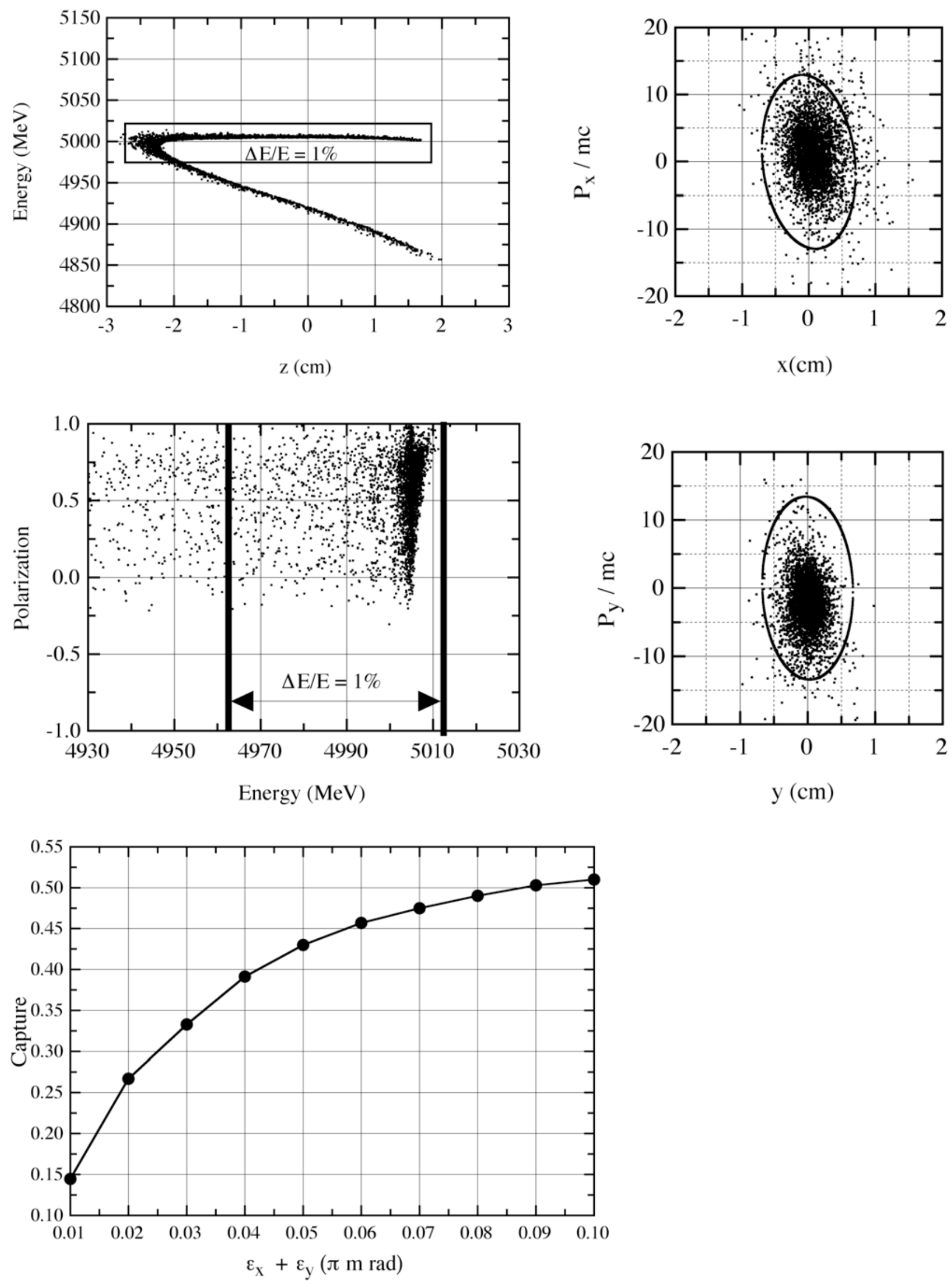

Fig. 5. Positron beam distribution after LTR. 

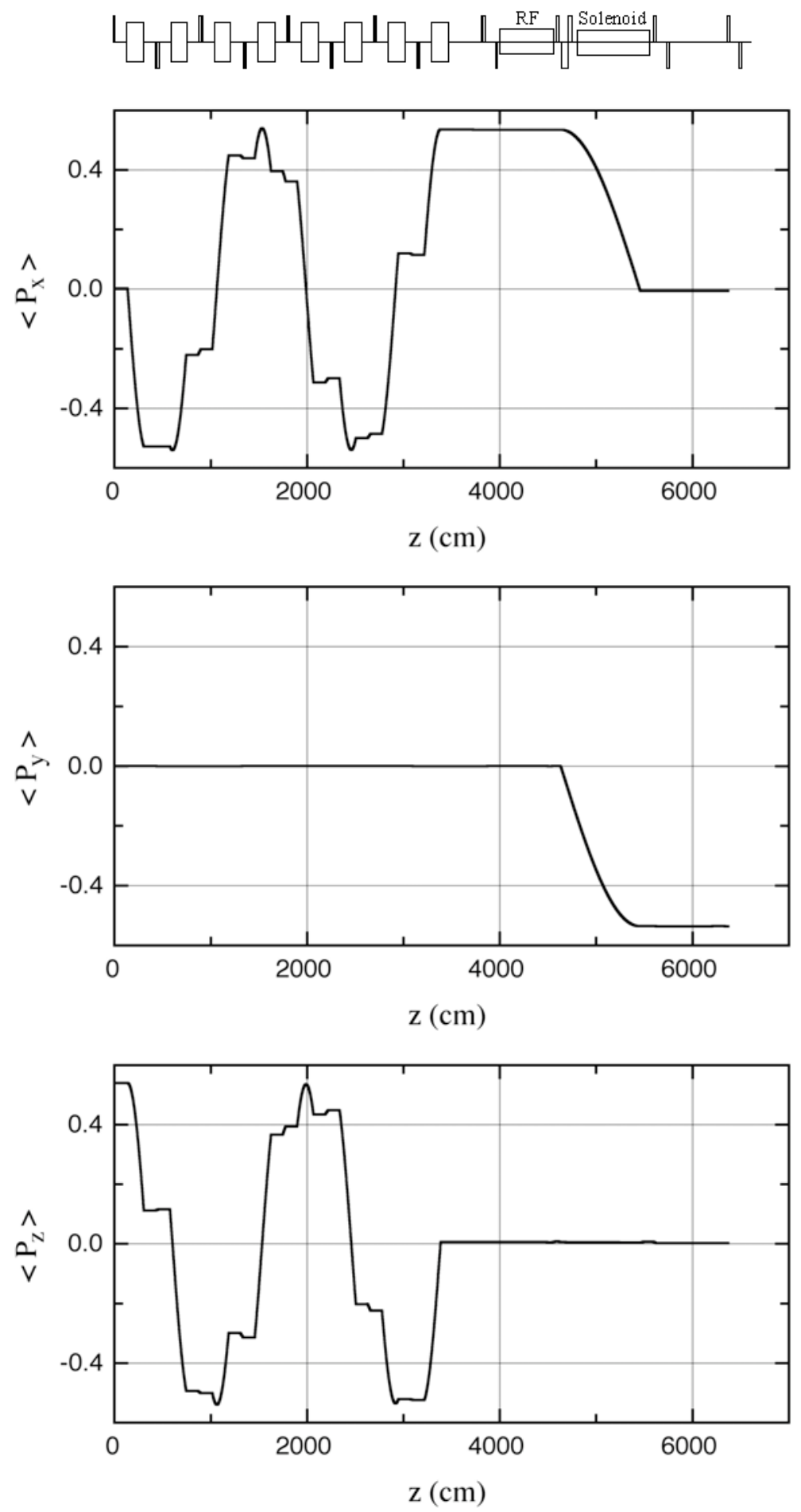

Fig. 6. Average beam polarization along the structure. 


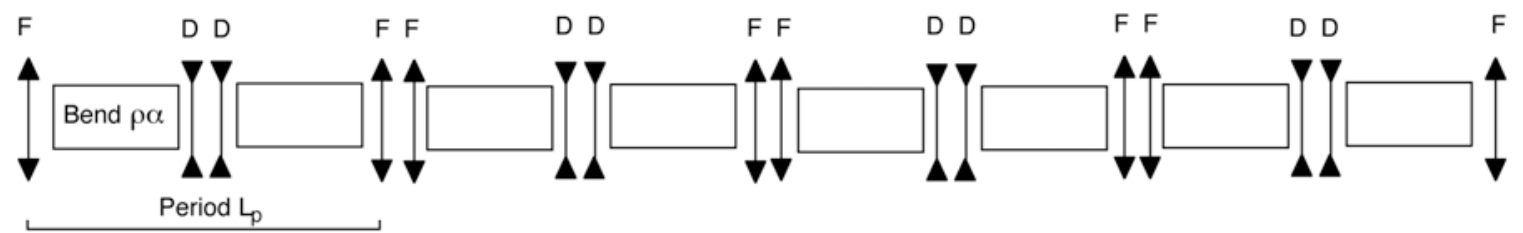

Fig. 7. Schematic layout of bending arc. 